\title{
Digital Data Acquisition for Gamma-Ray Spectroscopy
}

\author{
I. P. Etim, E. P. Inyang, and E. A. Thompson
}

\begin{abstract}
The performance characteristics of the digital pulse processor (DPP) as a low power pulse processor for gamma-ray semiconductor detector were analyzed. The FWHM energy resolution of the germanium detector was obtained as $0.35 \pm 0.5 \%$ and is in proper range of published value. The pulse resolution for peaking time of $0.8 \mu s, 3.2 \mu s$ and $8.0 \mu s$ was obtained to be $1.16 \pm 0.05 \%, 1.03 \pm 0.05 \%$ and $1.01 \pm 0.05 \%$ respectively. A good range was obtained for the residual energy at various energy peaks. This lie between $-2.3 \mathrm{KeV}$ and $1.3 \mathrm{KeV}$.The optimum peaking time was obtained at $6 \mu$ s where the FWHM goes through a minimum value from the plot of FWHM against peaking time. The dead time plot gave a linear fit for a peaking time of $3.2 \mu$ s and a curve at $19.2 \mu \mathrm{s}$.
\end{abstract}

Keywords - Gamma ray Spectroscopy; Nuclear structure; Digital data acquisition system; Instrumentation system.

\section{INTRODUCTION}

The Amptek Digital Pulse Processor (DPP) is a low power pulse processor for use with X-ray and gamma-ray semiconductor detectors. It digitalizes the preamplifier output signals, replacing both the shaping amplifier and Micro channel analyzer (MCA) in a traditional analogue spectroscopy system. Digital pulse processors, such as DPP, offer several advantages over traditional systems, including improved performance (very high resolution, reduced ballistic deficit, higher true output and enhanced stability), enhanced stability and low power consumption [1].

The signal that is supplied to the DPP is the preamplifier output. The DPP digitalizes the preamplifier output, applies real-time digital processing to the signal, detects the peak amplitude (digitally), and bins this value in its histogramming memory, generating an energy spectrum [2]. The spectrum is then transmitted over the DPP's universal serial bus (USB) interface to the user's computer. The DPP hardware is controlled over the USB interface, permitting the user not only to start and stop acquisition but to optimise shaping times, threshold levels, and other key parameters [3].

In this experiment, the DPP module contains a DP4 processor board which has been packaged in two different setups.In the first set up the DP4 module is mounted in a Nuclear instrument model (NIM) crate. The NIM front panel contains the signal in and signal out sockets. While in the second setup the DP4 module is mounted inside a PX4 module, which has an integrated power supply. In each case, the germanium detector preamplifier is connected directly to the DPP input.

\section{THEORY}

The DPP replaces many different components in a traditional instrumentation system: the shaping amplifier, the MCA, logic circuit and several auxiliary components [4]. Fig. 1 shows the block diagram of a traditional analogue nuclear instrument.

In addition to the detector and preamplifier, it includes separate modules for the shaping amplifier, an MCA, Power supplies, and auxiliary logic function.

In contrast, Fig. 2 shows the block diagram of an instrument based on digital pulse processing. It includes some basic elements and implements the same functions. The main difference is that the analogue to digital conversion occurs closer to the front of the processing chain, immediately after the preamplifier [5].The input to the digital processing core is the preamplifier output. This is digitalized, then real-time digital processing is applied to the signal, the peak amplitude is detected (digitally), and this value is binned in histogramming memory, generating an energy spectrum and then to the user's computer after passing

Submitted on December 05, 2021

Published on January 18, 2022.

I. P. Etim, Department of Physics, University of Calabar, Nigeria.

(e-mail: ini2etim@yahoo.com)

E. P. Inyang, Department of Physics, National Open University of Nigeria, Nigeria

(e-mail: etidophysics@gmail.com/einyang@noun.edu.ng)

E. A. Thompson, Department of Physics, University of Calabar, Nigeria.

(e-mail: edyy7000@yahoo.com) 
through a series of interface [6].

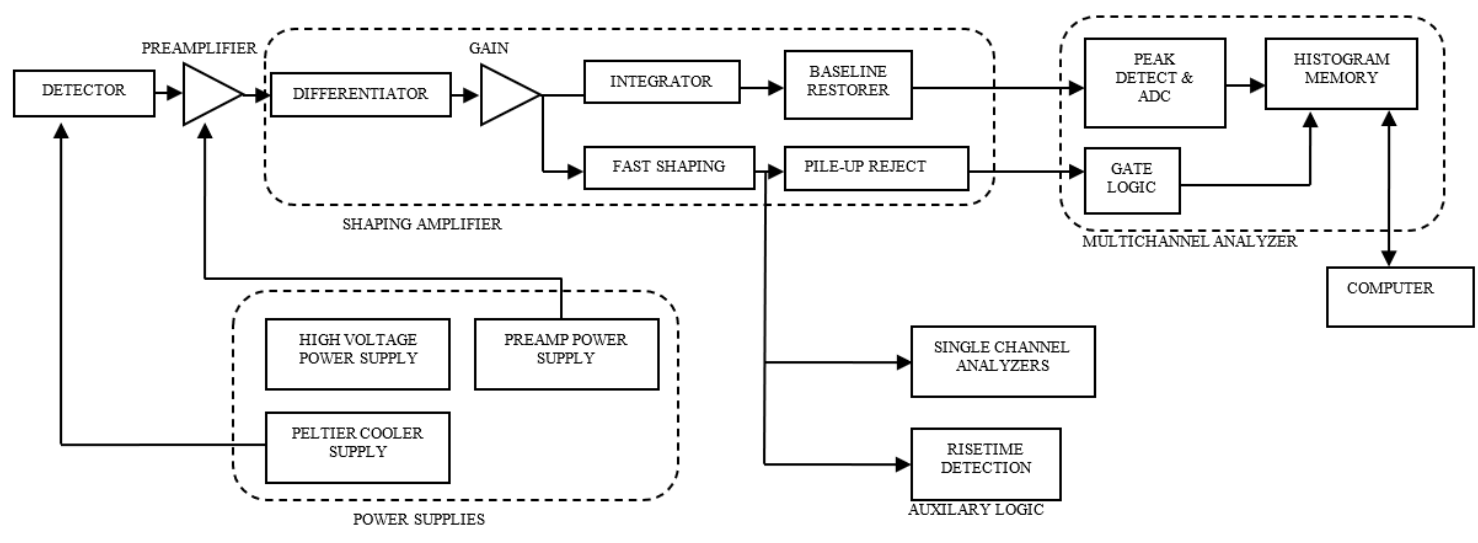

Fig. 1. Block diagram of a traditional analogue spectroscopy system.

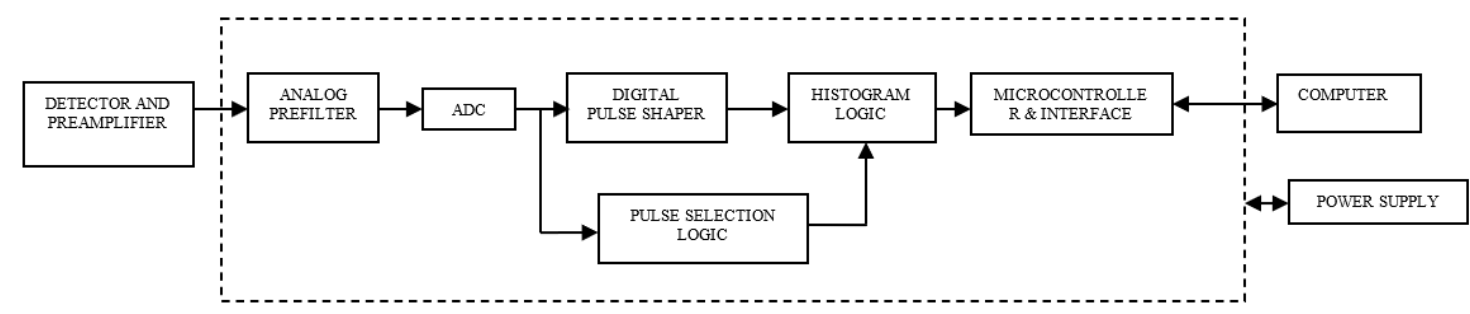

Fig. 2. Block diagram of the DP4 module. It includes the same functions as a traditional system, but in a single compact module.

In an analogue pulse processing system, the preamplifier output goes to a shaping amplifier and then to a MCA. The shaping amplifier's purpose is to permit an accurate determination of the peak height. The pulse shaping removes the direct current (DC) baseline, reduces distortion due to overlapping pulses, and filters out the broadband noise. The shaping amplifier also amplifies the pulse to permit accurate measurements. In the digital system, the pulse processor digitalizes the preamplifier output, then shapes the pulse with a digital filter running in the field programmable gate array (FPGA).The functionality is the same as the analogue system, but the digital pulse processor moves the digitalization earlier in the signal processing chain. The digital system allows more flexibility and greater performance by implementing these processing elements in software [7].

\section{A. Digital Shaping and Peaking Time}

With the DPP the user can control the peaking time parameter $T_{p}$ in a similar way that the pulse shaping time can be controlled in an analogue spectroscopy amplifier. A given peaking $T_{p}$ time will have a performance similar to that of an analogue amplifier with a shaping time given by $\tau$, where $\mathrm{T}_{\mathrm{p}}=2.2 \tau$. The analogue shaping time $\tau$ is determined by various parameters related to the filter and shaping stages of an analogue amplifier, including the time constant of the low pass filter stages, the pulse duration, noise bandwidth (in the frequency domain). Their relationship depends on the details of the transfer function of the pulse-shaping filter [8].

\section{B. Fast and Slow Channels}

The fast and slow channels are two parallel signal processing paths inside the DPP, operating at different shaping times, as shown in Fig. 4.The slow channel is used with a long shaping time constant, is optimized to minimize electronic noise, to obtain an accurate pulse height. The fast channel which has a short shaping constant is optimized to detect pulse which is closely spaced in time and so overlap in the slow channel. For most detectors, electronic noise is minimized by using a long shaping time constant, e.g using 20-50 $\mu$ s for a typical low noise Si detector. Therefore the slow channel is operated at this long time constant, and its output is connected to the peak detect circuit and used to obtain the energy spectrum [9].

Since radiation interacts in the detector at random intervals, it is possible to have two interactions occur within the processing time of the slow channel. Even at low count rates this occurs occasionally and in most applications, it is useful to operate at a high count rate to minimize data acquisition time. Two problems occur when the pulse overlap in time: only a single pulse is recorded rather than two, and the detected peak has incorrect amplitude. To address this, there is a fast channel with a peaking time of $0.4 \mu$ s. Pulses that overlap in the slow channel but not in this fast channel may be rejected from the spectrum, to minimize the distortions [10]. If pile-up reject (PUR) is turned on, then they are rejected. Furthermore, the fast channel is used to measure the true incoming count rate (ICR), where far fewer pulse is rejected. The fast channel 
usually has a much higher noise level and so the fast channel threshold is usually higher than the slow channel. The counts recorded by the DPP are those in the spectrum, ie. those in the slow channel. The incoming counts recorded by the DPP are those detected in the fast channel, where the dead time is much less.

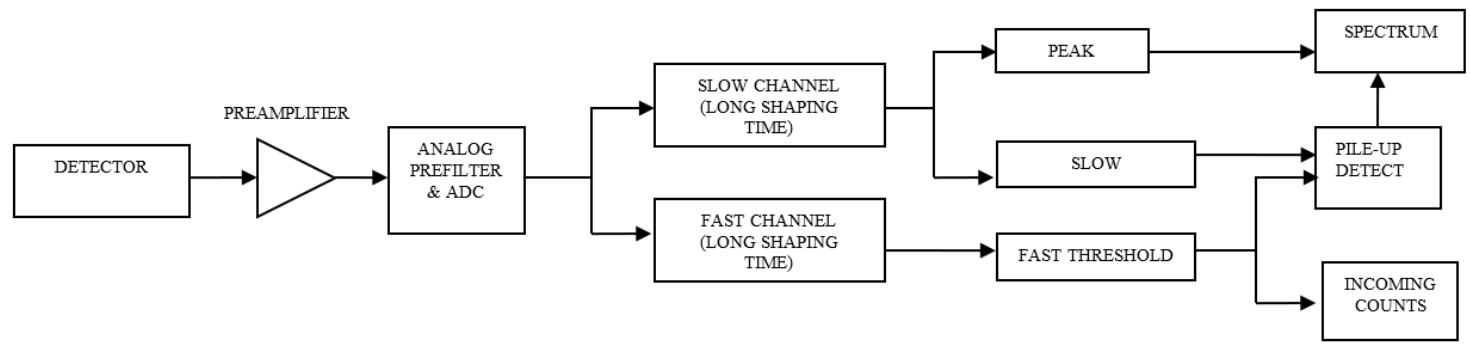

Fig. 3. Schematic of the fast and slow shaping channels in the DPP, each with a separate user-adjustable threshold.

\section{Dead Time Measurement}

The DPP system can count the real event rate from the detector using the fast channel since this has a very short shaping time and is not rate limited. If the fast threshold is correctly set the ADMCA software displays the number of true event as Input Counts [11], [12]. The slow channel records the actual measured number of events and displays it as Counts. In a dead time plot of measured count rate against true count rate, a linear data is obtained until a point where the dead time becomes significant and the measured count rate drops off rapidly.

\section{EXPERIMENTAL PROCEDURE}

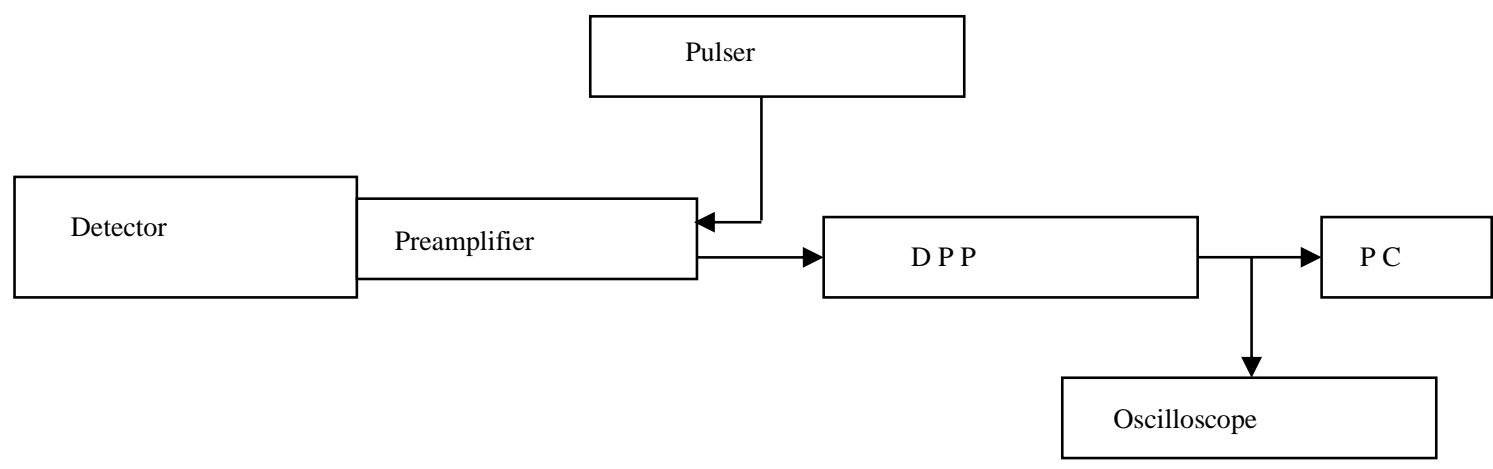

Fig. 4. Experimental set-up.

\section{A. Setting Up the DPP}

All connections were properly checked. The ADMCA program was initiated and connected to the DPP. The DPP configuration file was then loaded. The Ge detector preamplifier OUTPUT was then connected to the DPP input and the purser switched off. The threshold setting was optimized.

\section{B. Acquiring a Pulse Spectrum}

The pulser was switched on and a positive pulser spectrum was acquired for a peaking time of $3.2 \mu$ s. Two more pulse spectra were acquired with a peaking time of $0.8 \mu$ s and $8.0 \mu \mathrm{s}$. The pulser peak resolution was calculated for each peaking time and compared [13].

\section{1) Monitor signal}

The tektronic oscilloscope was used to measure the fall time of a decimated input pulse. The oscilloscope was selected from the monitor in order to view a copy of the trace on the computer screen. Using the oscilloscope the rise time and the flat top width of the pulse was measured. The results were compared with the DPP set up.

\section{Gamma Spectroscopy and Calibration 3.3.1 ${ }^{137}$ cs Spectrum}

A gamma-ray spectrum was acquired using a ${ }^{137} \mathrm{Cs}$ source. The full width at half maximum (FWHM) energy resolution of the detector was measured by defining the region of interest (ROI) around the photo peak.

\section{1) Energy Calibration}

A set of spectra was acquired using ${ }^{137} \mathrm{Cs},{ }^{60} \mathrm{Co}$ and ${ }^{22} \mathrm{Na}$. An energy calibration of the detector was carried 
out. A graph of the residual energy of each peak was plotted against peak energy.

2) Shaping Time and Noise Measurements

Using ${ }^{137} \mathrm{Cs}$ source a set of gamma-ray spectra was acquired for different values of digital shaping time. A graph of the FWHM resolution of the photopeak was plotted against the peaking time.

3) Dead Time Measurement

Using a peaking time of $3.2 \mu \mathrm{s}$. Five gamma sources $\left({ }^{137} \mathrm{Cs},{ }^{22} \mathrm{Na},{ }^{60} \mathrm{Co},{ }^{133} \mathrm{Ba}\right.$ and $\left.{ }^{241} \mathrm{Am}\right)$ were put in a metal case of the Ge detector and a set of spectra was acquired for 120 seconds. As the sources were removed one after the other and in each case, the true counts and measured counts were recorded. A dead time graph was plotted. The peaking time was then increased to $19.2 \mu \mathrm{s}$ and the same procedure was repeated.

\section{EXPERIMENTAL RESULTS}

The pulser peak resolution at Peaking time of $3.2 \mu$ s was $1.03 \pm 0.05 \%$. At $0.8 \mu$ s resolution was $1.16 \pm 0.05 \%$ and at $8 \mu$ s resolution was $1.01 \pm 0.05 \%$. Using the oscilloscope, the rise time was measured to be $8.32 \pm 0.42 \mu \mathrm{s}$ and the flat top width was $0.5 \pm 0.05 \mu \mathrm{s}$ and this is in agreement with the DPP set up of $8 \mu$ s for peaking time and $0.5 \mu$ s for flat top width. The FWHM energy resolution of the detector was $0.35 \pm 0.05 \%$.

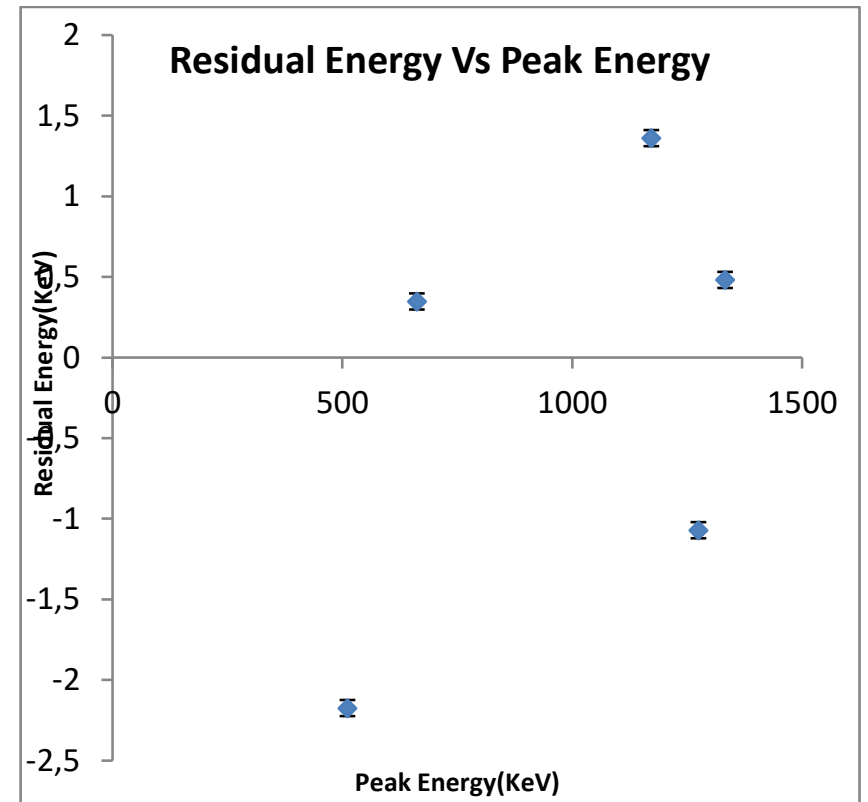

Fig. 5. Graph of Residual energy against Peak energy. The residual energy was obtained from the software after calibration was carried out as the difference between the measured energy and calibrated energy.

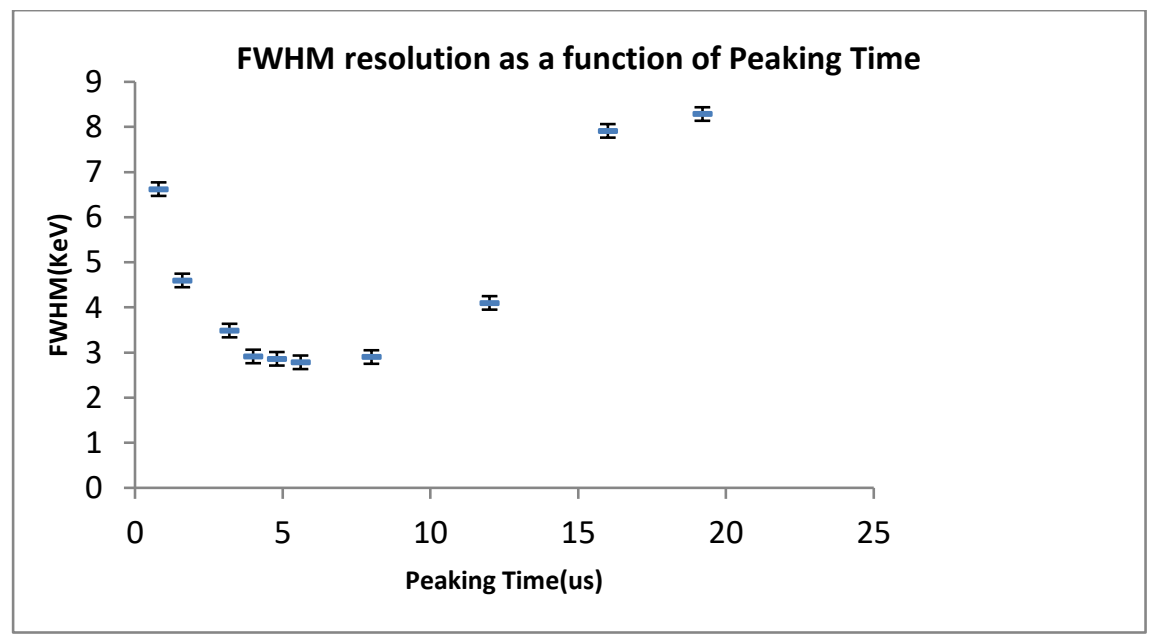

Fig. 6. Plot of FWHM against Peaking time to demonstrate the variation of noise with shaping time. 


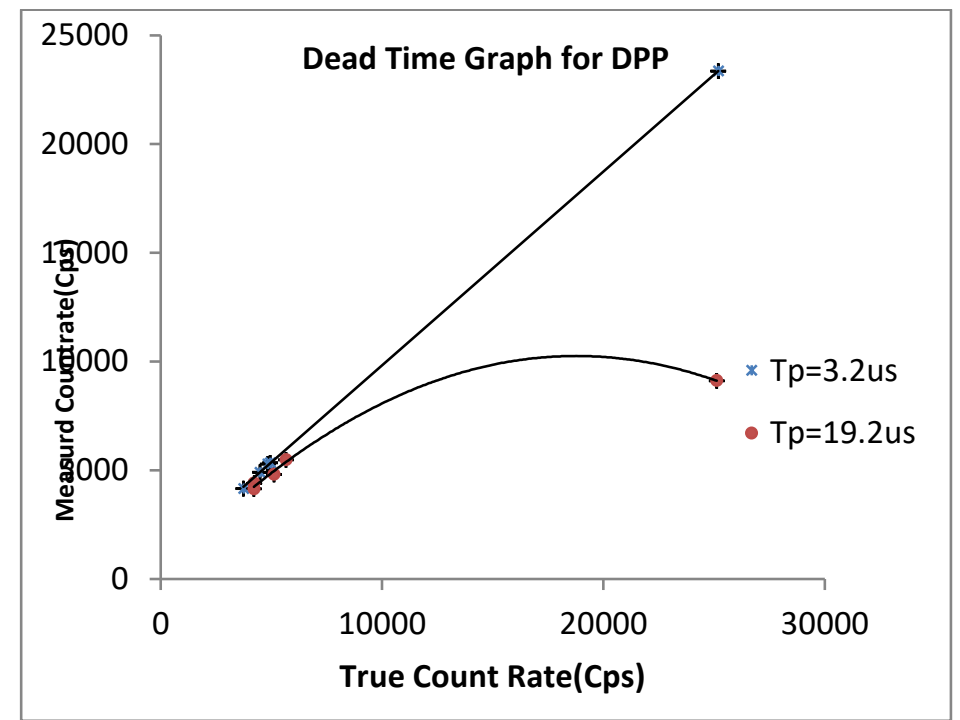

Fig. 7. Variation of measured count rate against the true count rate at two peaking times.

\section{DISCUSSION}

From the plot of residual energy against Peak energy. The range of energy is found to be between $2.3 \mathrm{KeV}$ and $1.3 \mathrm{KeV}$.There is an optimum peaking time that minimizes the FWHM of the measured gamma-ray peak from the detector. As the peaking time is increased from small values, the FWHM decreases (resolution improves). This is because the ballistic deficit decreases and the contribution of series noise (due primarily to fluctuations in the channel current of the input FET) is reduced. At the other extreme, increasing the peaking time causes a gradual increase in FWHM due to a greater sensitivity to parallel noise which originates from fluctuation in leakage current. At an optimum peaking time between these extremes, the FWHM goes through a minimum value of $6 \mu$ s as observed from Fig. 5.The effects of pile-up and baseline shift are important for all but the smallest values of peaking time near the left of the plot. For larger values of the peaking time, these effects increasingly contribute to the measured FWHM and are reflected in the steeper rise to the right of the minimum. In general, as the counting rate is increased the optimum peaking time moves to a smaller value and the effects of pile-up and baseline shift becomes severe.

The dead time graph is observed to be linear at a peaking time of $3.2 \mu \mathrm{s}$. But at $19.2 \mu \mathrm{s}$ the dead time becomes severe the graph becomes a curve. This shows that there is a strong dependence of the shape of the distribution on the peaking time. The measured count rate is expected to drop at a longer peaking time. A long shaping time introduces dead time but electronic noise is minimized. This is the reason the slow channel is operated at a longer peaking time in order to obtain accurate pulse height. At a peaking time of $19.2 \mu \mathrm{s}$, the pile-up rejection causes the measured count rate to pass through a maximum value as a function of true count.

\section{CONCLUSION}

The operation and functionality of the DPP have been assessed. The pulse peak resolution at different peaking time was calculated and the FWHM energy resolution of the germanium detector was obtained to be $0.35 \pm 0.05 \%$.The variation of residual energy and peak energy has been illustrated. A graphical relationship between FWHM and peaking time was used to discuss the noise of the system. The dead-time measurement was carried out from a plot of measured count against true count for two different peaking times. At lower peaking time a linear fit was obtained but at higher peaking time a curve was gotten with the measured count rate passing through a maximum value as a function of true count.

\section{ACKNOWLEDGEMENTS}

I. P. Etim would like to thank my partners Yit Fong and Suren K, the academics, postgraduate demonstrators and Gary Strudwick for their help and guidance in the lab.

\section{REFERENCES}

[1] G. F. Knoll, Radiation Detection and Measurement, J.Wiley \& Sons, York ,1989.

[2] ROOT Data Analysis Framework, https://root.cern.ch//. 
[3] K. Debertin and R.G.Helmer, Gamma and X-Ray Spectroscopy with Semiconductor Detectors, North-Holland, Amsterdam, 1988.

[4] Laboratory Script, RDI 21, Digital DAQ and gamma-ray spectroscopy, University of Surrey, 2009.

[5] E. P.Inyang, E. P. Inyang and E.S.William,Assessment of the exposure of radio frequency radiation from Wi-Fi routers in Calabar metropolis Nigeria. The Nigerian Journal of Scientific Research. 2017; 16(2):490-494.

[6] E. P. Inyang, E. P.Inyang, E.S.William, P.O.Ushie, and G. A. Oteikwu, Measurement of Electric Field Radiation from 11KVA High Tension Power Line and its Environmental Effects in Calabar Metropolis, Nigeria. Physical Science International Journal. 2018; 18(2):2348-0130.

[7] S .Das, S. Samanta, R. Banick, R.Bhattacharjee,K. Basu, R.Raut,S.S.Ghugre,A.K.Sinha, S. Bhattacharva and S. Imran, A Compton suppressed detector multiplicity trigger based digital DAQ for gamma-ray spectroscopy. Nuclear Instruments and methods in Physics Reseach Section A: Accelerators, Spectrometers, Detectors and Associated Equipment, 2018; 893:138-145.

[8] R. Palit, S. Saha, J. Sethi, T. Trivedi,S.Sharma,B.S. Naidu, S. Jadhav, R.Donthi, P.B.Chavan,H.Tan, and W. Hennig. A high speed digital data acquisition system for the Indian National Gamma Array at Tata Institute of Fundamental Research. Nuclear Instruments and methods in Physics Reseach Section A: Accelerators,Spectrometers, Detectors and Associated Equipment, 2012; 680:90-96.

[9] R.C.Pereira, J. Sousa, A.M.Fernandes,F.Patricio, B. Carvalho, A. Neto, C.A.F. Varandas, G Gorini, M. Tardocchi,D.Gin and A. Shevelev. ATCA data acquisition system for gamma-ray spectrometry. Fusion engineering and design, 2008; 83:341-345.

[10] L. Di-Wen, W.Hong-Yi,L.Zhi-Huan, X. Chuan, and J. Lin. Perormance of digital data acquisition system in gamma-ray spectroscopy. Nuclear Science and Techniques, 2021; 32(8):1-11.

[11] M.A.Riley,J.Simpson, and E.S.Paul, High resolution gamma-ray spectroscopy and the fascinating angular momentum realm of the atomic nucleus. Physica scripta, 2016; 91:123002

[12] E.P.Inyang, E.P.Inyang and M.B. Latif A Correlation: TL response of synthetic fused quartz with 60Co gamma (high dose) source and 90Sr/90Y Beta(low dose) source. Bulletin of Pure and applied Sciences-Physics, 2019; 38(1):06-12.

[13] R.S. Dong, L.Zhao, and J.J.Qin. Design of a 20-Gsps 12-bit time-interleaved analog-to-digital conversion system. Nuclear Science and Techniques, 2021; 32:18-25. 